\title{
One-Step Customized PEEK Cranioplasty After 3D Printed Resection Template Assisted Surgery for a Frontal Intraosseous Meningioma: A Case Report
}

\author{
Amaury De BARROS ${ }^{1}$, David BRAUGE ${ }^{1}$, Romain QUÉHAN¹, Zoe CAVALLIER², Franck-Emmanuel ROUX ${ }^{1,3}$, \\ Emmanuel MOYSE
}

${ }^{1}$ Toulouse University Hospital, Department of Neurosurgery, Toulouse, France

${ }^{2}$ Toulouse University Hospital, Department of Maxillofacial Surgery, Toulouse, France

${ }^{3}$ CNRS UMR5549 Brain and Cognition (Cerco), Hôpital Purpan, Toulouse, France

Corresponding author: Amaury De BARROS amaurydebarros@yahoo.fr

\section{ABSTRACT}

AIM: To present one-step customized cranioplasty for intraosseous meningiomas.

CASE DESCRIPTION: The authors report the case of a 54-year-old woman with a consequent frontal intraosseous meningioma invading the superior sagittal sinus. The patient only suffered from local pain and cosmetic damage. A complete resection was scheduled with a one-step reconstruction of the frontal bone by a polyetheretherketone (PEEK) specific implant. This implant was computer-assisted designed and manufactured and verified by the surgeon before the intervention. During surgery, the resection was guided by a computer designed resection template and by the classic neuronavigation system. Cranioplasty has been considered optimal intraoperatively by surgeons. The patient, a few weeks after surgery, underwent a subcutaneous fluid collection, rapidly resolutive with a circumferential pressure bandage. Six months after surgery, the patient considered the surgery a success with a very good cosmetic result and a total regression of her local pain.

CONCLUSION: One-step computer-assisted cranioplasty is a safe and effective procedure for large skull defects. PEEK specific implant for cranioplasty offer advantages compared to other materials that will be discussed under the scope of the one-step reconstruction.

KEYWORDS: Cranioplasty, Osseous meningioma, One-step reconstruction, CAD/CAM, PEEK

ABBREVIATIONS: CAD/CAM: Computer-assisted design / Computer assisted manufacturing, PEEK: Polyetheretherketone, PMMA: Polymethylmethacrylate, PSI: Patient specific implant, SSS: Superior sagittal sinus

\section{INTRODUCTION}

T ntraosseous meningioma or "osteomeningioma" are rare entities representing less than $1 \%$ of all meningiomas (12). Calvarial intraosseous meningioma is the most frequent (6), and is rarely revealed by neurological deficits, but often by skull deformations, leading to an unsightly appearance for patients. Most often these lesions are benign but resection can sometimes be extensive. As a consequence, cranioplasty with good cosmetic results can be challenging for the surgical team. Historically, many techniques with different materials have been used for cranioplasty, including autologous bone, titanium mesh, porous polyethylene, polymethylmethacrylate (PMMA) or hydroxyapatite $(10,15)$. Polyetheretherketone (PEEK) implants are now widely used for cranioplasty (1), cra-
Amaury De BARROS (D) : 0000-0001-9724-8616 David BRAUGE

Romain QUÉHAN
(D) : 0000-0001-5364-0640

(D) : 0000-0001-9392-9430
Zoe CAVALLIER

(D) : 0000-0001-5203-6767

Franck-Emmanuel ROUX (D) : 0000-0002-4209-7117

Emmanuel MOYSE (D) : 0000-0003-2731-263X 
niofacial defects repair (4), or others parts of skeleton reconstruction (8). PEEK prosthesis advantages are radiolucency, chemical inertness, robustness and comfort. It does not create artifacts on imaging and does not conduct temperature (10).

The recent advances in the era of computer-assisted design and computer-assisted manufacturing (CAD/CAM) prosthesis allow surgeons to consider the resection of large intraosseous meningioma with one-step reconstruction with a patientspecific implant (PSI) designed using a preoperative three dimensional computerized tomography (3D CT) scan.

This report describes the case of a woman suffering from frontal intraosseous meningioma treated by surgical resection

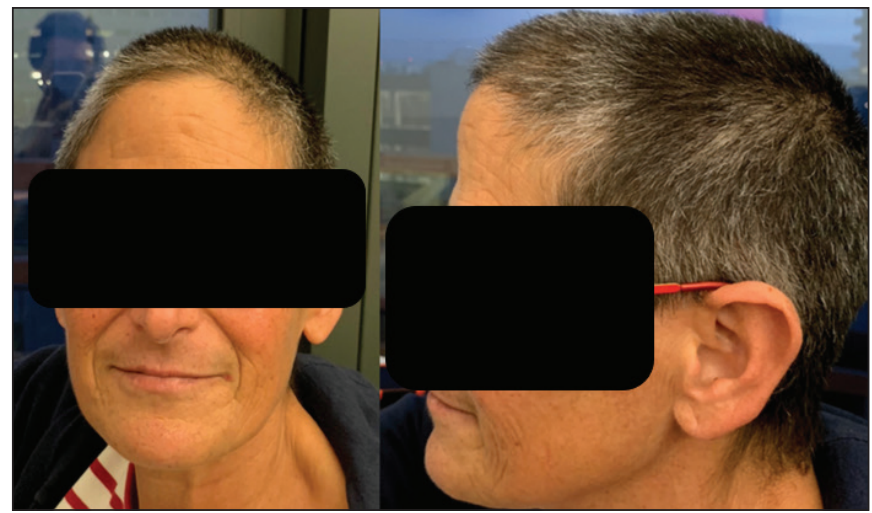

Figure 1: Photographic illustration of the cosmetic aspect of the frontal intraosseous meningioma in this 54-year-old woman. using a $3 D$ printed resection template modelled on the $3 D$ printed skull of the patient with a one-step reconstruction of the skull defect using a computer-assisted designed PEEK PSI.

The patient gave us her written consent for using her personal data for this report.

\section{CASE REPORT}

\section{History}

A 54-year-old patient consulted the neurosurgical unit for a slowly growing "bump" that began several years ago. There were no neurological elements in the medical history or physical examination. The patient's complaint was centered on frontal pain due to the mass effect on the scalp and the cosmetic damage due to the tumor (Figure 1). The scalp in the vicinity of the tumor was normal. All further investigations ruled out metastases, primary malignant tumor or a Paget disease.

\section{Initial Imaging}

A nonenhanced head CT-scan showed major hyperostosis originating from the frontal bone. In the coronal plane, the largest measure revealed a width of $77.7 \mathrm{~mm}$. The gadoliniumenhanced 3 tesla 3D T1 MRI revealed a dural part invading the superior sagittal sinus (SSS) in its anterior part (Figure 2A-E).

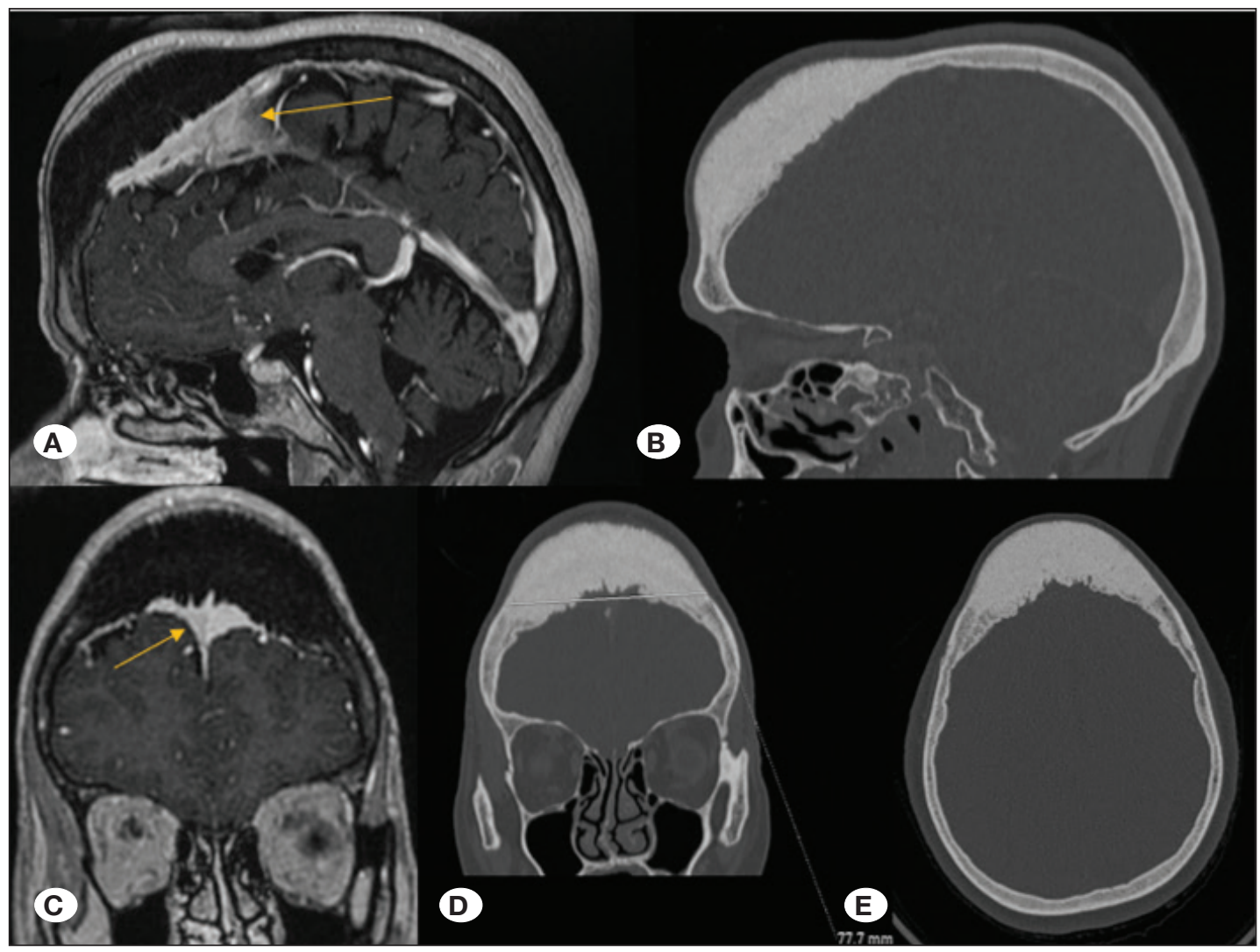

Figure 2: A) Gado-T1 MRI, sagittal plane. B) CT-scan, sagittal plane. C) Gado-T1 MRI, coronal plane. D) CT-scan, coronal plane. E) CT-scan axial plane. Yellow arrows represent the SSS invasion. 


\section{Presurgical Planning}

A 3D high resolution CT-scan with a matrix size of $512 \times 512$ $\mathrm{mm}$ with $1.0 \mathrm{~mm}$ slice thickness and no interslice gap of the patient was transferred to the manufacturing company (Stryker Corporation, Kalamazoo, MI 49002 U.S.A.). A volumetric segmentation of the meningioma was defined and resection margins were determined in accordance with the surgeon's guidance. A 3D printed skull was created and was used to elaborate in mirror the resection template (Figure 3A-C). After evaluation of the defect caused by meningioma resection and guided by the virtual estimation of the defect created (Figure 3D), PSI was manufactured to be as cosmetically suitable as possible with a $5 \mathrm{~mm}$ thickness (Figure 3E-F). The $\mathrm{PSI}$, resection template and $3 \mathrm{D}$ printed skull were sterilized and made available throughout the surgical procedure for comparison, verification or adjustment with patient and tumor reality.

\section{Surgical Treatment}

Prophylactic antibiotics (intravenous Cefazolin 2000 mg) were administered 30 minutes before incision. A coronal scalp and galeal incision was performed in order to widely expose the lesion (Figure 4A). With the help of the resection template (Figure 4B), resection margins defined in the presurgical planning were outlined with electrical bistoury (Figure 4A). Using a neuronavigation system (BrainLab; Munich, Germany) to manage the SSS, five burr holes were performed all around the lesion. After dural detachment, holes were connected with a craniotome and the osseous part of the meningioma was removed (Figure 4C). Dural invasion was carefully dissected, then resected controlled by neuronavigation system and the anterior third part of the SSS was removed after clipping. A duraplasty was performed with polyesterurethane (Neuropatch $^{\circledR}$, B.Braun Melsungen AG) in a tight fashion. PSI was placed at the level of the cranial vault defect and spatial conformation and immediate cosmetic result were checked before fixation with MatrixNEURO ${ }^{\mathrm{TM}}$ (DePuy Synthes Companies, Switzerland) (Figure 4D). Two dural tenting sutures were placed in the middle of the plasty to limit the risk of extradural detachment which could lead to compressive extradural hematoma. Scalp was closed in a two-layer fashion with surgical staples for the skin. Blood loss was estimated around $300 \mathrm{~mL}$ and the length of the intervention was $3 \mathrm{H} 35$ min.

\section{Postoperative Course}

No immediate post-procedure adverse events were encountered. Patient was free of neurological deficits, and classic postoperative pain was controlled by first line antalgic therapy. A control CT-scan with $3 \mathrm{D}$ reconstruction was performed 48 hours after surgery in order to control possible neurological damages, hematoma and cosmetic results. The PSI was estimated to be in a good placement and only a moderate pneumocephalus was described (Figure 5A-F). Anatomopathology revealed a $\mathrm{WHO}$ grade I meningioma.

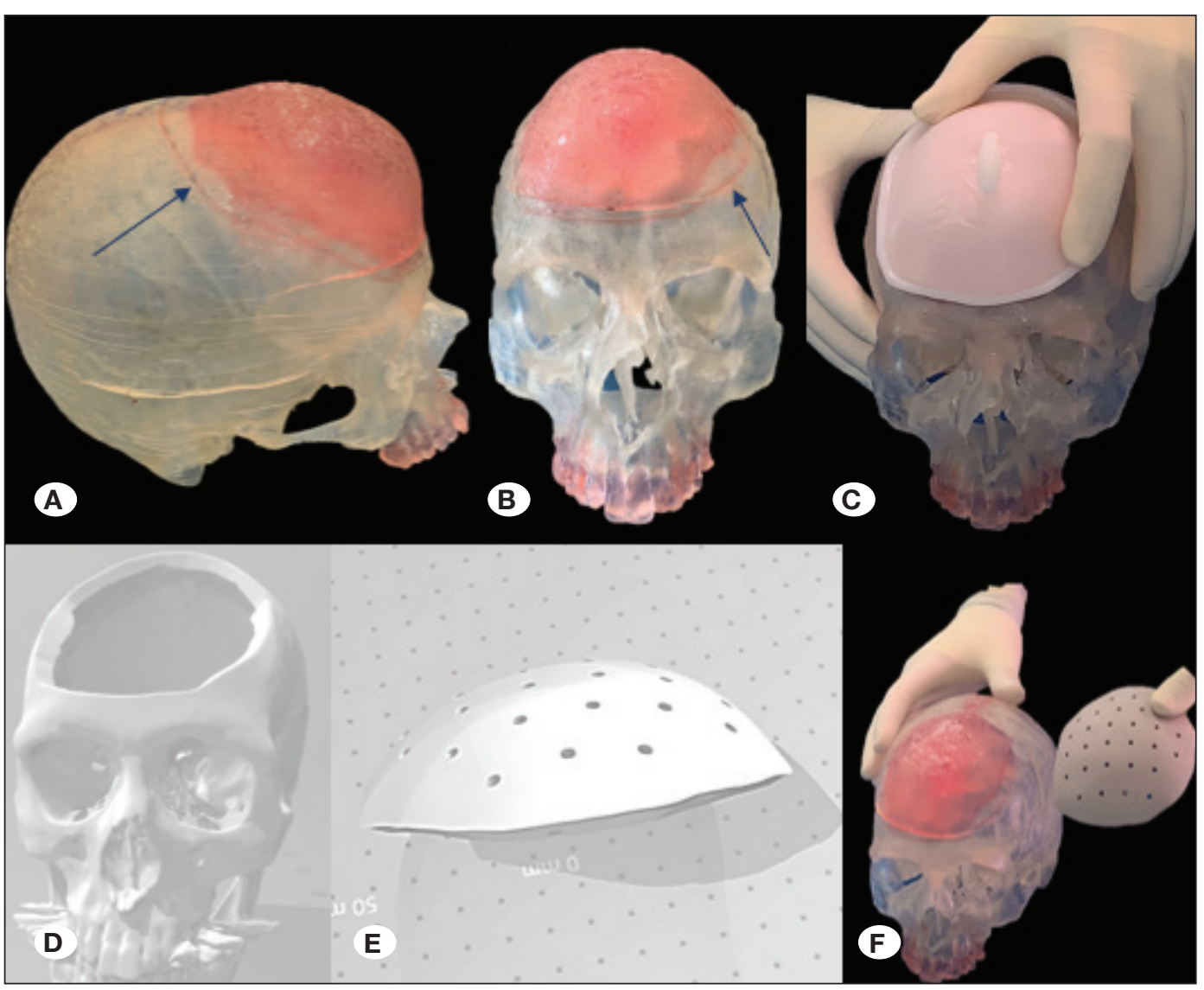

Figure 3: A) 3D printed skull, lateral view. B) 3D printed skull, frontal view. C) Resection template applied to the 3D printed skull. 3D printed skull acts as a negative for the creation of the resection template. D)Computer-assisted cranial vault defect estimation used for creation of the patient specific implant (PSI). E) Virtual aspect of the PSI. F) 3D printed skull with the definitive computerassisted manufactured PSI just before application to the patient. Blue arrows (a and b) represent margin of resection. 
De Barros A. et al: Computer-Assisted PEEK Cranioplasty

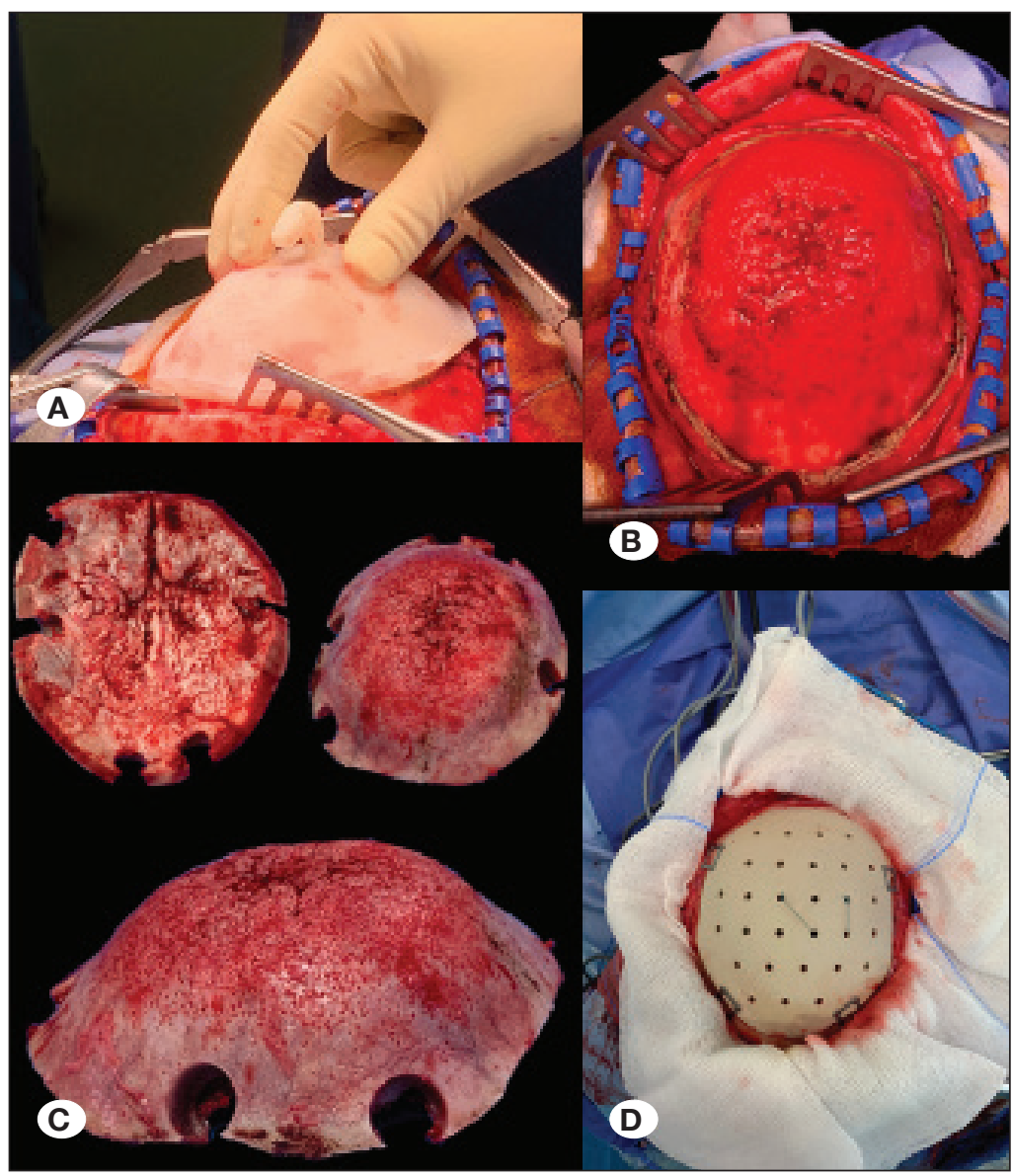

Figure 4: A) Application of the resection template in order to delineate the resection. B) Surgical exposure of the osseous meningioma. Resection margins are delineated with an electrical bistoury. C) The removed frontal osseous meningioma. D) The computerassisted manufacturing patient-specific implant placed and fixed into the cranial vault defect. The numerous prosthesis holes are used for adequate dural tenting sutures.

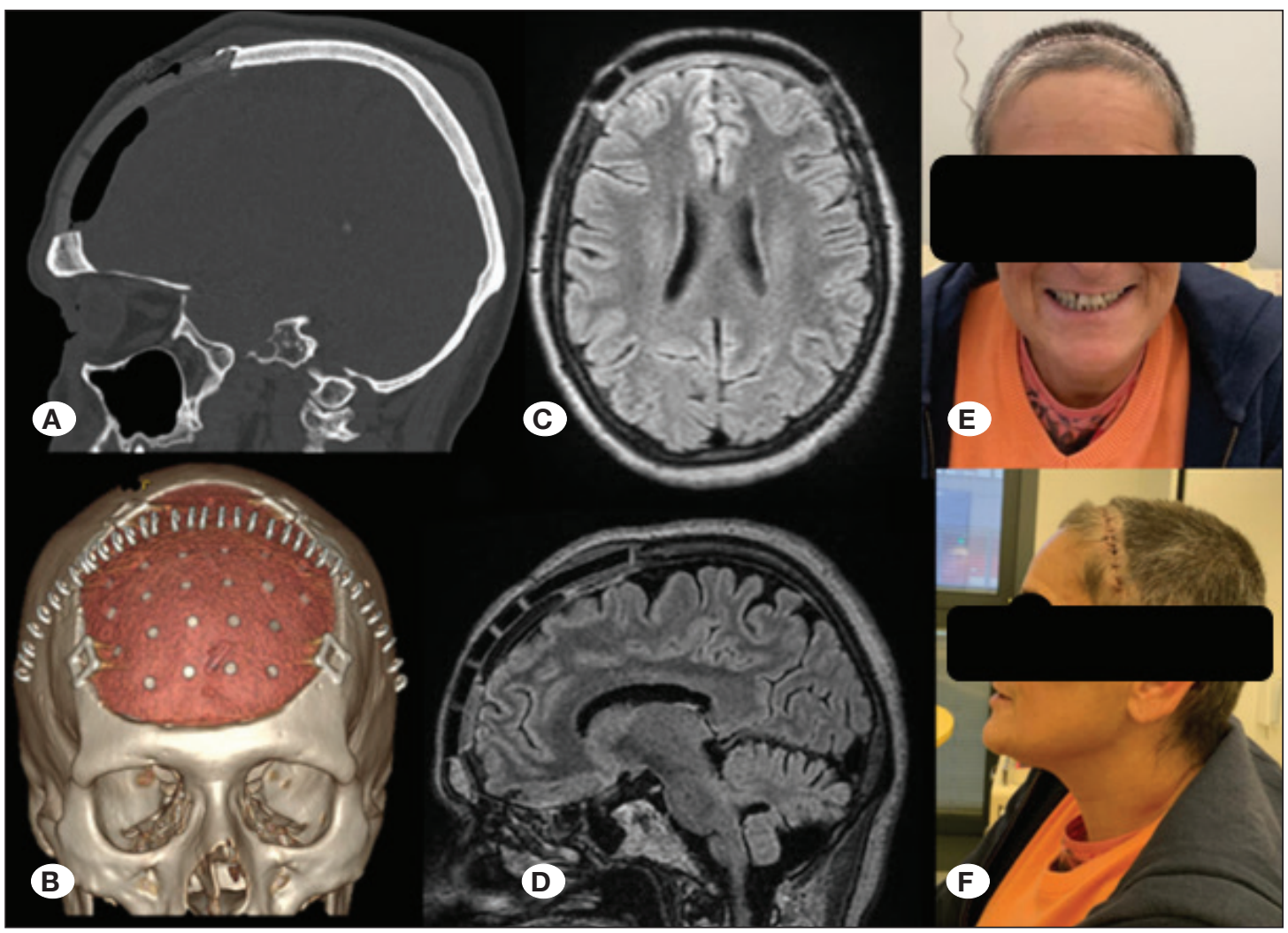

Figure 5:

A) Postoperative CT-scan (2 days after), sagittal view. B) Postoperative CT-scan, 3D volume rendering, frontosuperior view. C) Control MRI at 2 months, FLAIR, axial view. D) Control MRI at 2 months, FLAIR, sagittal view. E) Early postoperative aspect, frontal view. F) Early postoperative aspect, lateral view. 
Three weeks later, the patient developed a subcutaneous liquid collection favorized by surgical dissection without any local or general inflammatory signs. The surgeon decided to treat it with a circumferential pressure bandage for 6 weeks until the next consultation. Collection resolved and the patient was free of symptoms 3 months after the intervention, particularly the preoperative pain due to scalp traction. The cosmetic result was considered excellent by the patient herself. The control $\mathrm{MRI}$ at two months revealed a complete resection (Simpson grade I) with no particular complications and a total regression of pneumocephalus (Figure 5A-F).

\section{DISCUSSION}

Cranioplasty for a large cranial defect is still challenging for cranio-maxillo-facial surgeons and neurosurgeons. Most procedures are performed secondarily to the craniectomy, particularly when it is performed for refractory intracranial hypertension or for infectious reasons. CAD/CAM technology allows surgeons to perform one-step reconstructions, especially after large benign tumor resections without skin invasion, via manufacturing PSI. Many procedures have been developed until now using different methods of resections. Some authors described the use of a neuronavigation system with the fusion of the implant's virtual design for delineating accurate margins of resection (4). Others prefer to use a resection template (14) or the combination of both techniques (2). CAD/CAM technology can offer the possibility of printing a stereolithographic model (3D printed skull) and some authors have developed indirect methods with intermediary steps consisting of molding, via PMMA, the PSI directly to the $3 \mathrm{D}$ printed model $(3,11)$. With the exceptions of PEEK and PMMA, the other materials used until now for computer-assisted allografts have been hydroxyapatite or titanium.

In our experience, PEEK-specific implants are the best candidates for computer-assisted surgery due to their good possibility of conformation (they can also be drilled if needed) and their high mechanical resistance comparable to the cortical bone (7). Other qualities are their biocompatibility, comfort for the patient and radiolucency (10). Radiotherapy can be performed with the PEEK implant (4). Another important advantage is their thermostability. The PEEK implant can be sterilized and resterilized if needed, without deformations. This is of particular interest in the event of implant infection because they can be re-used after infection healing (5). Some disadvantages are the cost and the absence of osteointegration. The cost needs to be balanced with the reducing operative time or necessity of re-intervention compared to intraoperative molding procedures or two-step procedures, respectively. One of the main limitations after the cost was the presumed infectious risk, but two recent metaanalyses have declared that PEEK cranioplasties have a lower infection risk than autologous or PMMA cranioplasties $(9,13)$.

In comparison, titanium mesh offers the same mechanical resistance, cosmetic results and low infection rates but it can be deformed in case of trauma and it is a source of major radiological artifacts. A recent meta-analysis declared that titanium mesh presents more implant failure than PEEK prosthesis (9). Hydroxyapatite's main weakness is its fragility, sometimes leading to prosthesis fracture before osteointegration. Osteointegration is rarely rapid and total in the adult population compared to children, in our experience. PMMA is of good interest in small defects but its conformation, even with the help of a stereolithographic model, can be very challenging with regards to very large defects, leading to longer operative time and bleeding loss, which are risk factors for infection. In 2017, Broeckx et al. described a technique similar to ours; however, they designed and manufactured their PMMA-specific implant by themselves, considerably reducing the cost of the procedure (2). Their approach is very relevant for moderate defects, but requires a very good knowledge of CAD/CAM technology as well as availability of hardware material at one's institution. In our opinion, the collaboration of surgeons/engineers is very important and allows for completion of a complex PSI conformation that could not be reached alone. We hope that the development of a one-step reconstruction procedure will decrease the cost of implants.

\section{CONCLUSION}

A one-step surgical procedure using CAD/CAM technology in order to produce a patient-specific PEEK implant is a safe and accurate procedure with good cosmetic results and should reduce operative time and postoperative adverse events, particularly infection events. Further prospective investigations are needed to confirm this and strategies to reduce implant cost will be of particular interest in the future.

\section{REFERENCES}

1. Brandicourt $P$, Delanoé $F$, Roux FE, Jalbert $F$, Brauge $D$, Lauwers F: Reconstruction of cranial vault defect with polyetheretherketone implants. World Neurosurg 105:783-789, 2017

2. Broeckx CE, Maal TJJ, Vreeken RD, Bos RRM, ter Laan M: Single-step resection of an intraosseous meningioma and cranial reconstruction: Technical note. World Neurosurg 108:225-229, 2017

3. Guerrini F, Dallolio V, Grimod G, Cesana C, Vismara D, Franzin $A B$ : It is time to reduce free-hand manipulation: Case report of our proposal for an innovative 1-step cranioplasty. World Neurosurg 107:1052.e7-1052.e10, 2017

4. Jalbert F, Boetto S, Nadon F, Lauwers F, Schmidt E, Lopez R: One-step primary reconstruction for complex craniofacial resection with PEEK custom-made implants. J Craniomaxillofac Surg 42(2):141-148, 2014

5. Jonkergouw J, van de Vijfeijken SECM, Nout E, Theys T, Van de Casteele E, Folkersma H, Depauw PRAM, Becking AG: Outcome in patient-specific PEEK cranioplasty: A twocenter cohort study of 40 implants. J Craniomaxillofac Surg 44(9):1266-1272, 2016 
6. Lang FF, Demonte F: Primary extradural meningiomas: A report on nine cases and review of the literature from the era of computerized tomography scanning. J Neurosurg 93:11, 2000

7. Lethaus B, Safi $Y$, ter Laak-Poort M, Kloss-Brandstätter A, Banki F, Robbenmenke C, Steinseifer U, Kessler P: Cranioplasty with customized titanium and PEEK implants in a mechanical stress model. J Neurotrauma 29(6):1077-1083, 2012

8. Liu D, Fu J, Fan H, Li D, Dong E, Xiao X, Wang L, Guo Z: Application of 3D-printed PEEK scapula prosthesis in the treatment of scapular benign fibrous histiocytoma: A case report. J Bone Oncol 12:78-82, 2018

9. Punchak M, Chung LK, Lagman C, Bui TT, Lazareff J, Rezzadeh K, Jarrahy R, Yang I: Outcomes following polyetheretherketone (PEEK) cranioplasty: Systematic review and meta-analysis. J Clin Neurosci 41:30-35, 2017

10. Shah AM, Jung $H$, Skirboll S: Materials used in cranioplasty: $A$ history and analysis. Neurosurgical Focus 36(4):E19, 2014

11. Tan ETW, Ling JM, Dinesh SK: The feasibility of producing patient-specific acrylic cranioplasty implants with a low-cost 3D printer. J Neurosurg 1531-1537, 2016
12. Tokgoz N, Oner YA, Kaymaz M, Ucar M, Yilmaz G, Tali TE: Primary intraosseous meningioma: $\mathrm{CT}$ and $\mathrm{MRI}$ appearance. Am J Neuroradiol 26(8):2053-2056, 2005

13. van de Vijfeijken SECM, Münker TJAG, Spijker R, Karssemakers LHE, Vandertop WP, Becking AG, Ubbinck DT: Autologous bone is inferior to alloplastic cranioplasties: Safety of autograft and allograft materials for cranioplasties, a systematic review. World Neurosurg 117:443-452.e8, 2018

14. van de Vijfeijken SECM, Schreurs R, Dubois L, Becking AG, Becking LD, Karssemakers LHE, Milstein DMJ, van de Vijfeijken SECM, Depauw PRAM, Hoefnagels FWA, Vandertop WP, Kleverlaan CJ, Münker TJAG, Maal TJJ, Nout E, Riool $M$, Zaat SAJ: The use of cranial resection templates with $3 D$ virtual planning and PEEK patient-specific implants: A 3 year follow-up. Journal of Cranio-Maxillofacial Surgery 2018, (Online)

15. Zanotti B, Zingaretti N, Verlicchi A, Robiony M, Alfieri A, Parodi PC: Cranioplasty: Review of materials. J Craniofacial Surg 27(8):2061-2072, 2016 